\title{
Desafios com Palitos: Um Jogo Para o Ensino de Conceitos Específicos de Matemática
}

\author{
Wilk Oliveira dos Santos ${ }^{12}$, Daniel Borges F. da Silva ${ }^{2}$, Jário J. dos Santos Júnior ${ }^{2}$, \\ Ig Ibert Bittencourt ${ }^{2}$, Clovis Gomes da Silva Junior ${ }^{1}$
}

${ }^{1}$ Universidade de Pernambuco, Campus Garanhuns (UPE) Av. Cap. Pedro

Rodrigues, 105 - São José, CEP 55.294-902 - Garanhuns - PE - Brasil

${ }^{2}$ Núcleo de Excelência em Tecnologias Sociais (NEES) - Instituto de Computação Universidade Federal de Alagoas (IC-UFAL) Av. Lourival Melo Mota, S/N - Tabuleiro dos Martins, CEP 57072-900 - Maceió - AL - Brasil

\{wos, dbfs, jjsj, ig.ibert\}@ic.ufal.br, clovis.gomes@upe.br

Resumo: É sabido que a disciplina de Matemática é considerada uma das mais complexas do currículo escolar, sendo a principal responsável por reprovações de estudantes do ensino fundamental e médio, bem como que os jogos representam um importante mecanismo para auxiliar na resolução desta problemática. Neste sentido, este texto apresenta o jogo "Desafios com Palitos", um jogo para o ensino de conceitos específicos da Matemática (a saber, Raciocínio Logico, Algarismos Romanos e Conversão de valores). O texto apresenta ainda seu processo de desenvolvimento (por meio do processo de Virtualização de Jogos) e avaliação.

Abstract. We know the Mathematic is considered an more complex discipline in scholar curricula, be main responsible for disapproval of students in primary education and high school, as well, the educative games represent an important mechanism of auxiliary the resolution of the this problem. In this sense, this paper present a game "Desafios com Palitos", an game for teaching especially Mathematics concepts (for example: Logical Reasoning, Roman Numerals and Values Conversion). The paper present also the development process (through process Games Virtualization) and evaluate of game.

\section{Introdução}

É sabido que atualmente os jogos digitais estão cada vez mais presentes no dia a dia das pessoas de qualquer idade, como destaca Meira (2014). De acordo com Buchinger et al. (2012), os jogos eletrônicos estão ganhando cada vez mais espaço no mercado nacional e internacional despertando o interesse das instituições educacionais. Os atributos de tais jogos têm mostrado sua utilidade e eficácia para a educação no sentido da motivação e orientação para objetivos dos estudantes, mesmo depois de fracassar, como mostra Mattar (2010). Porém, de acordo com Albuquerque e Fialho (2009), nem todos os jogos educativos conseguem relacionar conteúdos educativos com o funcionamento do jogo.

Destaca-se também o fato de que, por vezes, os jogos educativos são chatos e sem noção, baseados em conceitos pedagógicos na qual não deveriam ser perceptíveis aos jogadores, em conformidade com Meira (2014). Contudo, vários estudos têm apoiado o uso de jogos para o ensino de disciplinas, com o objetivo de usar os aspectos 


\section{CBIE-LACLO 2015}

Anais dos Workshops do IV Congresso Brasileiro de Informática na Educação (CBIE 2015)

lúdicos dos jogos a fim de suprimir as dificuldades na aprendizagem de disciplinas consideradas complexas, como a Matemática.

Através dos jogos é possível desenvolver a linguagem, criatividade e raciocínio dedutivo (Silva Junior et al., 2006), que são exigidos na escolha de uma jogada bem como na argumentação durante a troca de informações, favorecendo, também, as atividades do dia a dia. De acordo com Rodrigues et al. (2010), vários modelos de desenvolvimento de software foram concebidos, onde alguns deles são específicos para o desenvolvimento de jogos, porém eles geralmente são superficiais e não se adaptam às necessidades dos jogos que incluam abordagens pedagógicas.

Neste sentido, este trabalho apresenta o jogo "Desafios com Palitos", um jogo para o ensino de conceitos específicos de Matemática, a destacar: raciocínio logico, algarismos romanos e conversão de valores. O jogo é composto por dez fases, definidas de acordo com os resultados de pesquisas (ver seção 2), e esta disponível para dispositivos móveis que executem as plataformas: Android, BlackBerry ou Windows Phone.

O jogo faz parte de um projeto de pesquisa, ensino e extensão realizado entre os anos de 2012 e 2015 por estudantes de graduação, mestrado e doutorado, além de professores de Matemática, bem como profissionais da Computação, Pedagogia e Psicologia da Universidade de Pernambuco e da Universidade Federal de Alagoas. O texto apresenta ainda seu processo de desenvolvimento baseado no processo de Virtualização de Jogos e sua avaliação.

Constatou-se que é importante trabalhar com uma equipe multidisciplinar, capaz de auxiliar no processo do desenvolvimento do jogo. Através da análise de desempenho, observou-se que o jogo teve a capacidade de reconhecer os toques e movimentos realizados pela maioria dos usuários. Analisando a interface da aplicação, concluiu-se que as cores foram utilizadas corretamente e os ícones relacionados aos botões foram facilmente entendidos (eg. Santos et al. (2014) e Santos et al. (2015)).

\section{Concepção da Ideia}

O jogo ora presentado faz parte de um projeto de extensão, pesquisa e ensino, realizado por uma equipe multidisciplinar nos últimos quatro anos, contando com colaboradores da Universidade de Pernambuco a da Universidade Federal de Alagoas, o projeto busca desenvolver objetos educacionais, capaz de proporcionar um processo de ensino e aprendizagem lúdico.

Para o desenvolvimento do jogo ora apresentado, inicialmente, foram realizadas entrevistas com docentes da disciplina de Matemática, além de profissionais da Pedagogia e Psicologia, com o intuito de levantar informações relevantes para decidir em que contexto o jogo se aplicaria, bem como os aspectos que poderiam ser abordados no mesmo, no que chamamos de analise de requisitos. Desta forma foi possível levantar uma discussão livre entre os docentes, na qual os mesmos expuseram suas concepções de forma colaborativa, de como deveria ser desenvolvido o jogo.

A escolha do jogo a ser desenvolvido partiu de um survey da literatura realizado por este grupo de pesquisa (eg. Santos e Silva Junior (2014)). Este trabalho teve como resultado o levantamento de oito jogos tradicionais, que foram usados 
outrora de forma positiva no ensino da Matemática, contudo entraram em processo de desuso em função dos avanços tecnológicos.

A etapa seguinte constituiu de uma análise de softwares semelhantes ao jogo proposto, levantados através da entrevista realizada com os docentes na etapa anterior. Como resultado, quatro jogos usados para esta comparação foram: (i) Puzzle de Fósforos ${ }^{1}$, (ii) Matchsticks ${ }^{2}$, (iii) Palitos: Racha a Cuca ${ }^{3}$ e (iv) Jogo dos Palitos ${ }^{4}$ (eg. Santos et al. (2014)). Os mesmos foram analisados considerando aspectos de usabilidade e processos cognitivos, destacando os princípios de atenção, memória e percepção, propostos por Preece et al., (2005). O Quadro 1 apresenta o resultado da análise, baseada nos elementos que compõem tais princípios.

Quadro 1. Análise dos jogos similares

\begin{tabular}{|c|c|c|c|c|}
\hline \multirow{2}{*}{ Elemento } & \multicolumn{4}{|c|}{ Jogo } \\
\cline { 2 - 5 } & (i) & (ii) & (iii) & (iv) \\
\hline Informações salientes & $\mathrm{X}$ & $\mathrm{X}$ & $\mathrm{X}$ & $\mathrm{X}$ \\
\hline Uso de cores para destacar palavras e figuras & $\mathrm{X}$ & $\mathrm{X}$ & $\mathrm{X}$ & $\mathrm{X}$ \\
\hline Informações apresentadas de forma sequenciada & $\mathrm{X}$ & $\mathrm{X}$ & $\mathrm{X}$ & $\mathrm{X}$ \\
\hline Interface sobrecarregada de informações & & & $\mathrm{X}$ & \\
\hline Faz o uso de ícones & $\mathrm{X}$ & $\mathrm{X}$ & $\mathrm{X}$ & $\mathrm{X}$ \\
\hline Faz o uso de efeitos sonoros & $\mathrm{X}$ & $\mathrm{X}$ & & \\
\hline
\end{tabular}

Em seguida realizou-se uma busca por trabalhos relacionados na literatura. Destaca-se o trabalho Bittar et al. (2010) por apresentar o processo de concepção do jogo Ludo Educativo destacando alguns desafios enfrentados ao desenvolver jogos para educação, e o trabalho Malfatti et al. (2010) por sua vez, tem como foco a construção de jogos com bons gráficos e que, ao mesmo tempo, exijam poucos recursos computacionais.

Tendo em vista todas as informações coletadas até este ponto da concepção da ideia do jogo, iniciou-se a etapa de desenvolvimento, visando desde a prototipação até a plataforma móvel escolhida.

\section{Desenvolvimento}

A etapa de desenvolvimento do jogo subdividiu-se em duas fases: $(i)$ prototipação e (ii) implementação. Em ambas as fases, buscou-se compreender processo a ser executado, realizando pesquisas com referenciais teóricos e operacionais com a finalidade de proporcionar o melhor resultado possível após executar esta etapa.

\subsection{Prototipação}

De acordo com Pressman (1995), o processo de prototipação capacita o desenvolvedor a criar um modelo de um software que será implementado. Para tal, as informações

\footnotetext{
${ }^{1}$ Mais informações consultar: http://migre.me/kjgM4

2 Mais informações consultar: http://migre.me/kjh4i

3 Mais informações consultar: http://migre.me/kjhaT

${ }^{4}$ Mais informações consultar: http://migre.me/kjhfF
} 
coletadas durante a concepção da ideia foram importantes para prosseguir nesta fase do desenvolvimento.

Algumas implicações de design foram estabelecidas considerando diferentes processos cognitivos, dos quais destacam-se ( $i$ ) atenção, focando nas fontes utilizadas nos botões, utilização de dicas, títulos e cores com a finalidade de evitar o acúmulo de informações causando um desconforto visual; (ii) percepção, focando nas posições dos botões de forma ordenada permitindo ao usuário descobrir rapidamente seu significado; e (iii) memória, focando na memorização dos elementos contidos na interface do jogo.

Por tratar-se de um jogo educativo, outro principio considerado na fase de prototipação, foi a Teoria da Carga Cognitiva que, segundo Nunes e Giraffa (2003), refere-se ao processamento humano de informações. Em outras palavras, é um conjunto de princípios que geram um ambiente de aprendizagem eficaz aumentando o processo de cognição do ser humano. A utilização desta teoria tem como objetivo tornar a interação humana mais alinhada ao processo cognitivo, concordando com Tarouco e Cunha (2006).

Para o desenvolvimento dos protótipos de tela foi utilizado o software Corel Draw X6. A escolha se deu em função da possibilidade de criar imagens com mais qualidade e fidedignas para que possam ser reutilizadas durante a fase de implementação do jogo.

\subsection{Implementação}

Nesta fase buscou-se identificar e analisar as engines ${ }^{5}$ (doravante chamadas motores) para desenvolvimento de jogos. Para isso, foram analisados quatro motores, baseandose nas informações obtidas nas etapas anteriores, avaliando qual tipo de jogo eles podem desenvolver e quais plataformas elas suportam (apresentados no Quadro 2), bem como a facilidade de programação do jogo (eg. Santos et al. (2015)).

Quadro 2. Análise de motores de desenvolvimento

\begin{tabular}{|c|c|c|}
\hline Motor & Tipos de jogos & Plataformas suportadas \\
\hline Game Maker $^{6}$ & 2D & Windows, Android, iOS, Ubuntu e Mac OS X \\
\hline${\text { Construct } 2^{7}}^{6}$ & 2D & Windows, Windows Phone, Android, iOS e BlackBerry \\
\hline Unity $^{8}$ & 2D e 3D & Windows, Windows Phone, BlackBerry 10, PS2, PS3 e Web \\
\hline UDK $^{9}$ & 2D e 3D & Windows, Android, iOS, Mac OS X, PS2, PS3 e PS4 \\
\hline
\end{tabular}

Baseado nas informações obtidas por meio desta pesquisa, foi decidido utilizar o motor de desenvolvimento Contruct 2, pelo fato de suportar o desenvolvimento para as plataformas Android, BlackBerry e WindowsPhone, além de possibilitar fazer o uso de programação drag-and-drop, facilitando o processo de programação do jogo. Um

\footnotetext{
${ }^{5}$ Softwares usados no desenvolvimento de jogos digitais, comumente chamados de "Motores" de Desenvolvimento de Jogos.

${ }^{6}$ Para mais informações, consultar: www.yoyogames.com

${ }^{7}$ Para maiores informações, consultar: www.scirra.com

${ }^{8}$ Para maiores informações, consultar: www.unity3d.com

${ }^{9}$ Para maiores informações, consultar: http://migre.me/kjCOr
} 
ponto importante desse motor é o fato de existir uma vasta documentação, na qual complementa o estudo de determinadas funções da ferramenta.

Neste sentido, o desenvolvimento do jogo seguiu um modelo de desenvolvimento "Iterativo", que permitiu um desenvolvimento dinâmico de cada uma das fases do jogo. Durante o processo de desenvolvimento, os profissionais da Computação, puderam consultar os demais profissionais envolvidos no trabalho, com o intuito de entender como implementar os aspectos lúdicos do jogo, atrelados aos aspectos pedagógicos necessários.

\section{O Jogo "Desafios com Palitos"}

Desafios com Palitos é um jogo tradicional que tem a capacidade de abordar tipos de conteúdos diferentes, como, por exemplo, Algarismos Romanos, Conversão de Valores e Raciocínio Lógico. O jogo pode ser utilizado como instrumento de ensino para a disciplina Matemática, utilizando tais tipos de conteúdos (Figura 1).

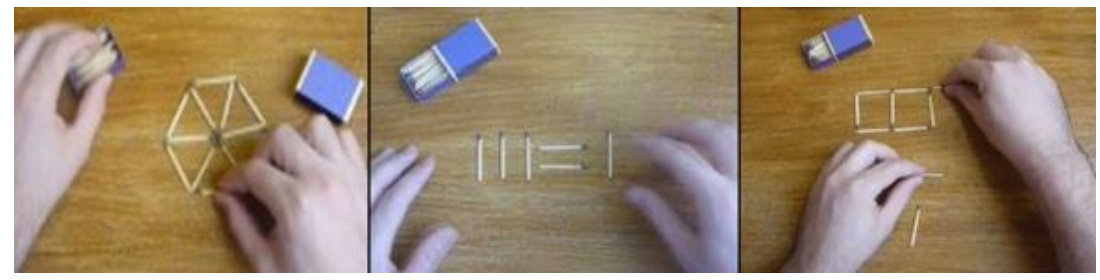

Figura 1. Jogo Desafios com Palitos tradicional

Utilizando as informações obtidas nas etapas anteriores, foi implementada a virtualização do jogo Desafios com Palitos. É importante destacar que já existem versões digitais deste jogo, tanto para desktop quanto para dispositivos móveis, mas elas carecem de aspectos relacionados à interface e aspectos pedagógicos (ver Sessão 2).

Com isso, foi desenvolvido um jogo, virtualizando o jogo tradicional, se preocupando com os aspectos de interface e pedagógicos, mencionados anteriormente, mantendo uma boa usabilidade sem perder a essência pedagógica. $\mathrm{O}$ jogo é composto por 10 fases e aborda assuntos como, por exemplo, conversão de valores, algarismos romanos e raciocínio lógico, dentre outros. A Figura 2 apresenta a tela inicial do jogo, bem como as fases 6 e 8 que tratam respectivamente raciocínio logico e figuras geométricas.
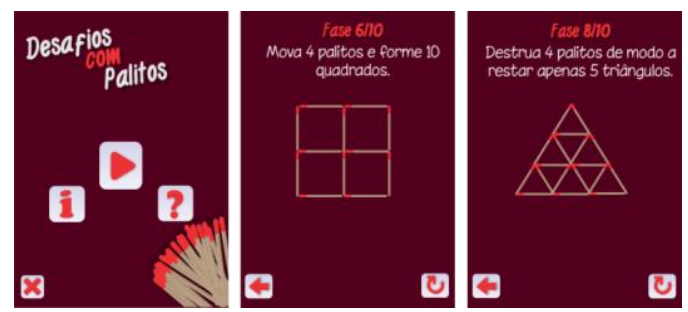

Figura 2. Exemplos de telas

Com o jogo implementado, iniciou-se a etapa de validação do software, com a finalidade de verificar se a aplicação está atendendo aos requisitos de interface. Tal etapa será descria na sessão seguinte. 
CBIE-LACLO 2015

Anais dos Workshops do IV Congresso Brasileiro de Informática na Educação (CBIE 2015)

\section{Avaliação}

Nesta etapa, buscou-se avaliar o jogo desenvolvido considerando os aspectos relacionados à interface da aplicação. Para isso, um grupo de 15 usuários, com idades entre 15 e 17 anos foram convidados para jogar livremente durante 20 minutos.

Em seguida, foi aplicado um questionário baseado na escala de Likert, onde foram apresentadas 7 assertivas a cerca do jogo, com a finalidade de colher opiniões dos usuários sobre o jogo. As respostas possíveis eram: concordo fortemente (CF), concordo (C), indiferente (I), discordo (D) e discordo fortemente (DF). As assertivas são mostradas à seguir, e o resultado do questionário é apresentado na Quadro 3.

- Assertiva 1: O jogo reconhece os meus toques na tela e movimenta corretamente os palitos;

- Assertiva 2: As cores do jogo são agradáveis;

- Assertiva 3: Entendi o que cada ícone representa;

- Assertiva 4: A interface possui poucas informações. As informações são de fácil entendimento;

- Assertiva 5: Entendi rapidamente o que deveria fazer nos desafios do jogo;

- Assertiva 6: Os desafios do jogo são motivadores e me deixa com vontade de continuar;

- Assertiva 7: Me senti satisfeito ao conseguir resolver os desafios.

Quadro 3. Validação do jogo

\begin{tabular}{|c|c|c|c|c|c|}
\hline \multirow{2}{*}{ Assertivas } & \multicolumn{5}{|c|}{ Escala de Likert } \\
\cline { 2 - 6 } & $\mathbf{C F}$ & $\mathbf{C}$ & I & D & DF \\
\hline Assertiva 1 & $36 \%$ & $55 \%$ & $9 \%$ & $0 \%$ & $0 \%$ \\
\hline Assertiva 2 & $46 \%$ & $18 \%$ & $18 \%$ & $18 \%$ & $0 \%$ \\
\hline Assertiva 3 & $46 \%$ & $27 \%$ & $19 \%$ & $9 \%$ & $0 \%$ \\
\hline Assertiva 4 & $27 \%$ & $55 \%$ & $18 \%$ & $0 \%$ & $0 \%$ \\
\hline Assertiva 5 & $30 \%$ & $30 \%$ & $10 \%$ & $20 \%$ & $10 \%$ \\
\hline Assertiva 6 & $45 \%$ & $44 \%$ & $11 \%$ & $0 \%$ & $0 \%$ \\
\hline Assertiva 7 & $46 \%$ & $36 \%$ & $9 \%$ & $9 \%$ & $0 \%$ \\
\hline
\end{tabular}

\section{Considerações Finais}

Após realizar o processo do desenvolvimento do jogo, percebeu-se que é possível virtualizar jogos tradicionais mantendo os mesmos princípios, pedagógicos $\mathrm{e}$ psicopedagógicos, atrelados às mecânicas contemporâneas dos jogos digitais. Destacase a importância da colaboração de uma equipe multidisciplinar durante $o$ desenvolvimento desse tipo de aplicação.

Através do processo de validação do jogo, foi possível notar que o mesmo reconheceu os toques e movimentos da interface da maioria dos usuários, e que as cores e elementos da interface foram utilizados corretamente, de uma forma que a maioria dos usuários não sentiram dificuldades em reconhecer as ações dos botões. Percebeu-se, também, que os desafios propostos por cada fase do jogo foram compreendidos.

Objetiva-se, como trabalhos futuros, desenvolver novas fases, com o propósito de tornar o jogo mais duradouro e motivador para os usuários, bem como executar novas avaliações com novos cenários e novos públicos. Uma versão (desktop) de teste para deste jogo pode ser encontrada no seguinte link: http://migre.me/rfnwB 


\section{Referências}

Albuquerque, R. \& Fialho, F., (2009) "Concepção de jogos eletrônicos educativos: Proposta de processo baseado em dilemas". In: Anais do XVIII Simposio Brasieliro de Jogos e Entretenimento Digital (SBGames). Rio de Janeiro - RJ.

Bittar, T.J., Lobato, L.L., Pereira, P.F. \& Longo, E., (2010) “Considerações para jogos educativos na Web com base nas experiências e resultados do desenvolvimento do Ludo Educativo". In: Anais do XIX Simposio Brasieliro de Jogos e Entretenimento Digital (SBGames). Florianopolis - SC.

Buchinger, D., Silva Hounsell, M.d. \& Dias, C., (2012). "Colaboratividade em um Jogo Eletrônico para Ensino sobre Dengue”. In Anais do XXIII Simpósio Brasileiro de Informática na Educação (SBIE). Rio de Janeiro - RJ.

Carissimi, A., (2009) "Virtualização: Princípios básicos e aplicações". Minicurso da $9^{a}$ Escola Regional de Alto Desempenho-ERAD, pp.39-69.

Malfatti, S.M., Guimarães, W.B., Simões, F.O. \& Brancher, J.D., (2010) "Desenvolvimento de Jogos Educacionais Voltados ao Projeto UCA". In: Anais do XIX Simposio Brasieliro de Jogos e Entretenimento Digital (SBGames). Florianopolis - SC.

Mattar, J., 2010. Games em educação: como os nativos digitais aprendem. São Paulo: Person Prentice Hall.

Meira, S., 2014. Cadê os jogos educacionais móveis? [Online] Available at: HYPERLINK "http://boletim.de/silvio/cad-os-jogos-educacionais-mveis/" http://boletim.de/silvio/cad-os-jogos-educacionais-mveis/ [Accessed 9 July 2014].

Nunes, M. \& Giraffa, L., 2003. A educação na ecologia digital. PPGCC/FACIN, PUCRS.

Preece, J., Rogers, Y. \& Sharp, H., 2005. Design de interação. Bookman.

Pressman, R.S., 1995. Engenharia de Software. São Paulo: Pearson Makron Books.

Rodrigues, H.F., Machado, L.d.S. \& Valença, A.M.G., (2010) "Definição e Aplicação de um Modelo de Processo para o Desenvolvimento de Serious Games na Área de Saúde”. In: Anais do XXX Congresso da Sociedade Brasileira de Computação (CSBC). Belo Horizonte $-\mathrm{MG}$.

Santos, W. O.; Silva Junior, C. G. (2014). "Uso de Jogos no ensino da Matemática: Uma análise entre os jogos tradicionais e os jogos digitais, baseada em pesquisa e mapeamento dos materiais encontrados na Web". In: Anais do X Seminario de Jogos Eletronicos, Educação e Comunicação (SJEEC). Salavdor - BA.

Santos, W. O.; Souza, A. A.; Oliveira, M. L.; Silva, A. P.; Tenório, A. K.; Rodrigues, A. N.; Junior, C. G. S. (2014) "Desafios com Palitos: Processo de Desenvolvimento de um Jogo Educativo". In: Anais do XIII Simposio Brasileiro de Jogos Digitais (SBGames). Porto Alegra - RS.

Santos, W. O., Silva Neto, S. R., Silva Junior, C. G. (2015). "Processo de Virtualização de Jogos para Uso como Mecanismo de Apoio ao Processo de Ensino e Aprendizagem da Disciplina de Matemática”. In: Anais do XI Seminário Jogos Eletrônicos Educação e Comunicação (SJEEC). Salvador - BA, p. 109-117. 
Anais dos Workshops do IV Congresso Brasileiro de Informática na Educação (CBIE 2015)

Santos, W. O., Silva Neto, S. R., I., I., Bittencourt, Silva Junior, C. G. (2015) "Avaliação de Jogos Educativos: Uma Abordagem no Ensino de Matemática". In: Anais do XXVI Simpósio Brasileiro de Informática na Educação (SBIE). Maceió - AL.

Silva Junior, C.G., Laurentino, O.J. \& Silva, R.F., 2006. O dominó como ferramenta para o ensino da matemática. Encontro Pernambucano de Educação Matemática (EPEM).

Tarouco, L.M.R. \& Cunha, S.L.S., 2006. Aplicação de teorias cognitivas ao projeto de objetos de aprendizagem. Revista Novas Tecnologias na Educação (RENOTE). v. 4 n. 2 . 